\title{
Acute pancreatitis and diabetes mellitus: a review
}

\author{
Allyson Richardson ${ }^{1}$ and Walter G. Park ${ }^{2}$
}

Departments of ${ }^{1}$ Internal Medicine and ${ }^{2}$ Gastroenterology and Hepatology, Stanford University Medical Center, Stanford, CA, USA

Received: September 14, 2020 Accepted: November 2, 2020

\section{Correspondence to}

Walter G. Park, M.D.

Department of Gastroenterology and Hepatology, Stanford University Medical Center, 300 Pasteur Drive, Endoscopy, Ho206B, Stanford, CA 94305 , USA

Tel: $+1-650-723-4102$

Fax: $+1-650-723-5488$

E-mail: wgpark@stanford.edu https://orcid.org/0000-0001$8187-4188$
Diabetes following acute pancreatitis (AP) is becoming increasingly recognized. It is unclear what subtype of diabetes mellitus (DM) occurs; however, type $3 \mathrm{C}$ diabetes mellitus $\left(\mathrm{T}_{3} \mathrm{CDM}\right)$ is gaining increasing recognition. $\mathrm{T}_{3} \mathrm{CDM}$ has differing pathophysiology than other subtypes of DM and therefore differing disease course and treatment. Current studies have examined the incidence and prevalence of DM following AP, and meta-analyses have shown around 15\% develop $\mathrm{DM}$ at 1 year with an increasing proportion developing DM at 5 years. It has been observed that some patients have transient hyperglycemia following AP episode with a subset developing persistent impaired glucose metabolism; however, the exact timeline is not well defined. The data on risk factors for developing DM after AP is limited and mixed; however, it is likely that severity of AP may impact the propensity to develop DM. Screening guidelines have not been established following AP; however, screening 1-year post-event will likely capture a sizable proportion of newly developed DM. The endocrine and exocrine pancreas are closely linked, and studies have found significant overlap in dysfunction of both after AP. Finally, there are some data to suggest that diabetes predisposes patients to structural changes in the pancreas and increased risk of developing AP.

Keywords: Pancreatitis; Diabetes mellitus; Incidence

\section{INTRODUCTION}

Acute pancreatitis (AP) is common, and over the past decade there has been a trend towards increased number of admissions, but lowered mortality $[1,2]$. Specifically, in the United States, AP is responsible for 250,000 admissions each year and has shown an increase in $20 \%$ of admissions over the past 10 years [3]. The vast majority (80\%) of admissions are mild, self-limited disease; however, long term consequences are still present [4]. One of those complications is endocrine dysfunction, and specifically impaired glucose metabolism or diabetes.

Diabetes is prevalent and its burden is felt worldwide. According to the World Health Organization, it affects around 422 million adults worldwide [5]. Type 2 diabetes mellitus (T2DM) is the most common sub-type; however, more and more recognition has been given towards other sub-types, namely diabetes related to disorders of the exocrine pancreas.

Diabetes of the exocrine pancreas or type $3 \mathrm{c}$ diabetes mellitus ( $\left.\mathrm{T}_{3} \mathrm{cDM}\right)$ is increasingly common and also under-recognized by providers [6]. One study found it to be more prevalent than type 1 diabetes mellitus ( $\mathrm{T} 1 \mathrm{DM})$ [7], and $\mathrm{T}_{3} \mathrm{CDM}$ accounts for $5 \%$ to $10 \%$ of diabetes in the western population [8]. Furthermore, there is a well-established relationship between diabetes and chronic pancreatitis [9] as well as pancreatic cancer $[9,10]$ but there is more and more emerging evidence for the association of diabetes with AP [11].

The goal of this review is to: summarize the existing literature on prevalence, natural history, risk factors of impaired glucose metabolism after AP; to explore the relationship with exocrine insufficiency; to discuss the potential bi-directional relationship between diabetes 
and AP; as well as to discuss the role of screening, diagnosis and treatment of diabetes in this cohort.

\section{DIABETES OF THE EXOCRINE PANCREAS}

Though it is not well established what sub-type of diabetes mellitus (DM) develops after AP, special consideration should be given to diabetes of the exocrine pancreas. Diabetes of the exocrine pancreas, otherwise known as T3cDM or "secondary pancreatic diabetes," is an established clinical entity that is often under-recognized [11]. In a 2017 study, looking retrospectively at population level data from England, diabetes following pancreatic disease was more common than T1DM. Upon further analysis, the vast majority of those cases (87.8\%) were identified as $\mathrm{T}_{2} \mathrm{DM}$ by clinicians [7]. T3cDM is more commonly characterized in the setting of chronic pancreatitis and pancreatic cancer as well as cystic fibrosis, hemochromatosis and prior pancreatic surgery [9]. It is less well characterized in AP, though still thought to occur [11].

Classification of $\mathrm{T}_{3} \mathrm{CDM}$ is important, as the proposed pathophysiology for T3cDM differs from TiDM and T2DM. The proposed mechanism involves inflammation, fibrosis, and sclerosis of pancreatic endocrine tissue (including cells that secrete glucagon, somatostatin, and pancreatic polypeptide), which leads to a reduction in total number of insulin producing islet cells and alteration of their function [11]. T3cDM affects all cells in the islets of Langerhans and therefore has features of both insulin resistance and insulin deficiency. Furthermore, several additional hormones are affected including glucagon, pancreatic polypeptide, incretin, adipokines (in the AP episode) leading to a unique clinical entity. This is characterized by a patient who has risk for hyperglycemic and hypoglycemic events with increased insulin requirements early in the disease course, but decreased risk of diabetic ketoacidosis [11].

The long-term management also differs in T3cDM. One study followed patients for up to 13 years and differentiated impaired glucose metabolism into T2DM and $\mathrm{T}_{3} \mathrm{CDM}$. They found that all of the patients who had $\mathrm{T}_{3} \mathrm{CDM}$ eventually required insulin, where as those diagnosed with $\mathrm{T} 2 \mathrm{DM}$ were predominantly controlled by oral medications [12]. This observation supports the proposed mechanism that $\mathrm{T} 3 \mathrm{cDM}$ is due to inflammation, scarring and islet loss, leading to less insulin secretion, rather than predominant insulin resistance found in $\mathrm{T}_{2} \mathrm{DM}$.

\section{DIAGNOSTIC CRITERIA}

The diagnosis of $\mathrm{T}_{3} \mathrm{CDM}$ has been difficult to distill. Currently similar diagnostic criteria for T2DM exist including: clinical symptoms of hyperglycemia and glucose of $\geq 200 \mathrm{mg} / \mathrm{dL}$ or asymptomatic individuals with at least two abnormal biochemical tests: fasting glucose $\geq 126 \mathrm{mg} / \mathrm{dL}, 2$-hour glucose $\geq 200 \mathrm{mg} / \mathrm{dL}$ after 75 -g oral glucose ingestion, or hemoglobin $\mathrm{Arc}(\mathrm{HbAlc}) \geq 6.5 \%$ [13]. However, the role of pancreatic dysfunction in diagnosis of $\mathrm{T}_{3} \mathrm{cDM}$ remains controversial. Many etiologies exist leading to $\mathrm{T}_{3} \mathrm{cDM}$ including: acute and chronic pancreatitis, pancreatic cancer, cystic fibrosis, hemochromatosis, etc. [11]. This makes including criteria based on pancreatic dysfunction and etiology difficult given the heterogeneity in disease and variable progression to T3cDM. For example, patients post-pancreatectomy may have an abrupt onset of $\mathrm{T}_{3} \mathrm{CDM}$, whereas those with AP may have more subtle, slower progression.

Others have proposed targeting the characteristics specific to T3cDM which included: impaired beta cell function, lack of insulin resistance, deficiency of lipid-soluble vitamins A, D, E, and K, and impaired release of glucagon-like peptide-1 and pancreatic polypeptide [11]. Specifically, Ewald and Bretzel [14] proposed the following diagnostic criteria: (all of the following must be met)

- A diagnosis of diabetes mellitus

- Evidence of exocrine pancreatic insufficiency (fecal elastase $1[\mathrm{FE} 1]<200 \mu \mathrm{g} / \mathrm{g}$ or abnormal direct function testing)

- Abnormal pancreatic imaging (endoscopic ultrasound, magnetic resonance imaging, and computed tomography)

- Absence of TiDM associated autoimmune markers (antibodies against glutamine acid decarboxylase, islet cell antigen, or insulin) [14].

These criteria have undergone criticism for being particularly difficult to implement clinically [15]; however, they provide a potentially more specific approach to di- 
agnosing T3cDM.

Finally, another study measured baseline and poststimulation insulin and $\mathrm{C}$ peptide levels as a distinguishing marker for insulin resistance versus beta cell destruction. Amongst the small number of patients in the study, they found a trend towards lower C peptide and insulin levels in those who had severe AP (compared to mild disease); however, they also found an increase in C peptide and insulin levels in those who developed $\mathrm{DM}$ in general [16]. In general, the wide range of diseases that lead to $\mathrm{T}_{3} \mathrm{cDM}$ and the variable timeline of disease development makes it difficult to have clear cut diagnostic criteria. Currently, it is favored to first establish a diagnosis of $\mathrm{DM}$, and then to pay particular attention to a patient's pre-disposing conditions, namely, disease of the pancreas, to determine if their pathology more closely aligns with $\mathrm{T}_{3} \mathrm{cDM}$ versus other subtypes (T1DM or T2DM). Careful delineation of $\mathrm{T}_{3} \mathrm{CDM}$ from other subtypes is important to ensure optimal follow-up and treatment [7].

\section{INCIDENCE/PREVALENCE}

As a self-limiting disease, the concept that AP could lead to diabetes is one that is of increasing interest. Several studies have attempted to characterize the incidence and/or prevalence of new onset diabetes following a single AP event. These studies range from small, single-center cohorts to population level data to meta-analyses (Table 1) [12,16-48]. The incidence and prevalence vary widely in these studies. These differences likely stem from study design bias including but not limited to variable follow-up periods and tertiary referral bias.

An important study worth explicitly noting was a meta-analysis conducted in 2014 that showed an incidence of DM after 1 year of $15 \%$ and up to $23 \%$ after 5 years. This study specifically looked at incidence following the first episode of AP. Furthermore, the investigators subdivided impaired glucose metabolism into pre-DM, $\mathrm{DM}$, and DM treated with insulin and found a pooled prevalence of $16 \%, 23 \%$, and $15 \%$, respectively over their study period [17].

Another meta-analysis conducted in 2019 showed a similar pooled incidence of $23 \%$ [18]. Upon further sub-analysis, the investigators found the pooled inci- dence within 5 years was 20\% and after 5 years was 37\%, showing a trend for increased development of endocrine dysfunction over time. This analysis, compared to the 2014 meta-analysis, used many of the same studies and also included additional studies up to 2017. Though there was heterogeneity in the data, it was similar to the previous meta-analysis and provides compelling evidence of incidence of DM following AP.

Other studies, however, show that DM is less common. Specifically, a large population-based study conducted using a national database in Taiwan showed only $5 \%$ developed endocrine dysfunction over a greater than 1 year follow-up period [19]. Various other studies show a wide range of incidence/prevalence. These studies have variable sample sizes, follow-up intervals and patient demographics, and exclusion criteria. Furthermore, some studies combined pre-diabetes and diabetes, whereas others separated these values. Finally, as physicians underrecognize T3cDM, the diagnostic criteria used in these studies varied making it difficult to compare results [6].

Given the data collected so far, it is difficult to ignore that a significant proportion of patients develop some form of impaired glucose metabolism after an episode of AP. The large meta-analyses have consistently shown close to a quarter of patients are diagnosed with diabetes at the 5-year mark, with the potential for even a greater portion after 5 years. Further studies are needed with strict inclusion and exclusion criteria as well as further characterization of what subtype of DM develops, to better characterize impaired glucose metabolism in this cohort.

\section{NATURAL HISTORY}

It is a known phenomenon that hyperglycemia occurs after critical illness [49]. When the body is under stress, especially in the setting of acute illness, it releases cortisol which stimulates gluconeogenesis in the liver and limits the uptake of glucose in the peripheral tissues leading to relative insulin resistance [50]. A study examined this phenomenon and found following Intensive Care Unit stay, stress induced hyperglycemia occurred in $17 \%$ of patients but only $4.8 \%$ of patients went on to develop T2DM. AP, leads to a similar acute illness and 
Table 1. Characteristics of studies examining DM after acute pancreatitis episode

\begin{tabular}{|c|c|c|c|c|c|c|}
\hline Study & Year & Study design & $\begin{array}{l}\text { Sample } \\
\text { size }\end{array}$ & $\begin{array}{c}\text { Proportion with } \\
\text { DM }\end{array}$ & $\begin{array}{c}\text { Mean } \\
\text { follow-up } \\
\text { period, mo }\end{array}$ & $\begin{array}{l}\text { Included } \\
\text { in meta- } \\
\text { analysis }\end{array}$ \\
\hline Das et al. [17] & 2014 & $\begin{array}{l}\text { Systematic review/ } \\
\text { meta-analysis }\end{array}$ & 1,102 & $\begin{array}{l}15 \% \text { (at } 12 \mathrm{mo} \text { ), } 23 \% \\
\text { pooled prevalence }\end{array}$ & & \\
\hline Zhi et al. [18] & 2019 & $\begin{array}{l}\text { Systematic review/ } \\
\text { meta-analysis }\end{array}$ & 13,894 & $23 \%$ (pooled) & & \\
\hline Johansen et al. [25] & 1972 & Prospective cohort & 22 & $4(18 \%)$ & 24 & 1,2 \\
\hline Olszewski et al. [26] & 1978 & Prospective case control & 25 & $7(28 \%)$ & 12 & 1,2 \\
\hline Seligson et al. [27] & 1982 & Prospective cohort & 9 & $2(22 \%)$ & 63 & 1,2 \\
\hline Angelini et al. [28] & 1984 & Prospective cohort & 19 & $1(5 \%)$ & $25,40^{\mathrm{a}}$ & 1,2 \\
\hline Eriksson et al. [29] & 1992 & Prospective cohort & 36 & $19(53 \%)$ & 74 & 1,2 \\
\hline Doepel et al. [30] & 1993 & Prospective cohort & 37 & $20(54 \%)$ & 74 & 1,2 \\
\hline Angelini et al. [31] & 1993 & Prospective cohort & 118 & $9(8 \%)$ & 53 & 1,2 \\
\hline Malecka-Panas et al. [32] & 1996 & Retrospective cohort & 47 & $8(16 \%)$ & $48-84$ & 2 \\
\hline Appelros et al. [33] & 2001 & Prospective cohort & 35 & $15(43 \%)$ & 83 & 1,2 \\
\hline Malecka-Panas et al. [34] & 2002 & Prospective cohort & 82 & $15(16 \%)$ & 56 & 1,2 \\
\hline Ibars et al. [35] & 2002 & Prospective cohort & 55 & $6(11 \%)$ & $1,6,12^{\mathrm{a}}$ & 1,2 \\
\hline Halonen et al. [36] & 2003 & Prospective cohort & 145 & $68(47 \%)$ & 66 & 1,2 \\
\hline Boreham et al. [37] & 2003 & Prospective cohort & 23 & $4(17 \%)$ & 3 & 1,2 \\
\hline Szentkereszty et al. [38] & 2004 & Prospective cohort & 22 & $3(14 \%)$ & 38 & 1,2 \\
\hline Hochman et al. [39] & 2006 & Prospective cohort & 25 & $8(19 \%)$ & $24,36^{\mathrm{a}}$ & 1,2 \\
\hline Kaya et al. [40] & 2007 & Prospective cohort & 112 & $13(21 \%)$ & 12 & 1,2 \\
\hline Yasuda et al. [41] & 2008 & Prospective cohort & 41 & $16(39 \%)$ & 56 & 1,2 \\
\hline Gupta et al. [42] & 2009 & Prospective cohort & 30 & $6(20)$ & 31 & 1,2 \\
\hline Pelli et al. [43] & 2009 & Prospective cohort & 46 & $5(11 \%)$ & 23 & 1.2 \\
\hline Andersson et al. [16] & 2010 & Prospective cohort & 39 & $9(23 \%)$ & 45 & 1,2 \\
\hline Uomo et al. [44] & 2010 & Prospective cohort & 38 & $6(16 \%)$ & 179 & 1,2 \\
\hline Garip et al. [45] & 2013 & Retrospective cohort & 96 & $33(34 \%)$ & 32 & 2 \\
\hline Vujasinovic et al. [22] & 2014 & Retrospective cohort & 100 & $14(14 \%)$ & 32 & 2 \\
\hline Chandrasekaran et al. [46] & 2015 & Prospective cohort & 35 & $17(48 \%)$ & 26.2 & 2 \\
\hline Ho et al. [19] & 2015 & Retrospective cohort & 12,284 & $618(5 \%)$ & $>24$ & 2 \\
\hline Winter Gasparoto et al. [47] & 2015 & Retrospective cohort & 16 & $5(31 \%)$ & 34.8 & 2 \\
\hline Lee et al. [24] & 2016 & Retrospective cohort & 3,187 & $324(10 \%)$ & 3.21 & \\
\hline Umapathy et al. [20] & 2016 & Retrospective cohort & 73 & $33(45 \%)$ & $>12$ & 2 \\
\hline Vipperla et al. [23] & 2016 & Retrospective cohort & 101 & $28(28 \%)$ & $34 \cdot 5$ & 2 \\
\hline Nikkola et al. [12] & 2017 & Prospective cohort & 47 & $7(15 \%)$ & 126 & 2 \\
\hline Tu et al. [48] & 2017 & Retrospective cohort & 113 & $34(30 \%)$ & 42.9 & 2 \\
\hline Tu et al. [21] & 2018 & Retrospective cohort & 256 & $154(60.2 \%)^{b}$ & 42.9 & \\
\hline
\end{tabular}

DM, diabetes mellitus.

${ }^{a}$ Authors followed-up different study populations for different lengths of time.

${ }^{\mathrm{b}}$ Included impaired glucose tolerance with DM. 


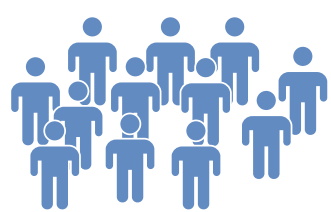

Acute pancreatitis episode

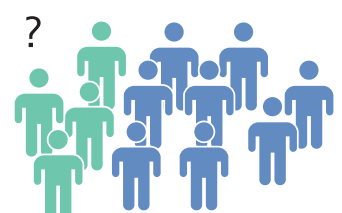

Transient hyperglycemia

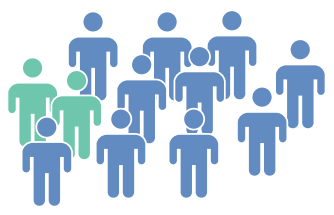

Diabetes
Mechanisms:

Inflammation

Scarring and fibrosis

Islet cell loss

Figure 1. Figure representing proposed natural history of diabetes mellitus following acute pancreatitis.

thus interferes with short- and long-term impaired glucose metabolism. This study exemplifies the complex natural history of DM in patients with critical illness and possibly identifies a similar process to what occurs following AP. It specifically highlights the distinction between transient hyperglycemia following critical illness and the development of chronic impaired glucose metabolism or diabetes.

In another study using a large population database in Taiwan, investigators evaluated the risk of developing DM after a first episode of AP. They found there were greater "odds" of developing insulin resistance in the first 3 months post-event (hazard ratio [HR], 5.9) compared to after 3 months (HR, 2.54). This demonstrates a previously discussed phenomenon of transient hyperglycemia post-event, followed by a smaller proportion going on to develop sustained impaired glucose metabolism [51].

In another study that included a cohort of severe AP patients, $45 \%$ developed new onset diabetes after their first episode of AP, with the vast majority developing it during the index admission [20]. In fact, the mean time from index admission to diagnosis of DM was 1 month. Development of DM was associated with the extent of necrosis in this study and brings up the question whether more severe AP episodes leads to more rapid development of $\mathrm{T}_{3} \mathrm{CDM}$.

These studies highlight that the time course and natural history of diabetes following AP is not clearly defined. It appears to be on the orders of months to years, with a trend for increasing disease prevalence farther from the index AP episode (Fig. 1). This may suggest that the injury associated with AP may not be entirely self-limiting. Rather the injury may set in motion an inflammatory process with subsequent fibrosis with ongoing implications towards endocrine insufficiency. More research should be done to further delineate this time course, so surveillance in groups at higher risk of developing DM can be pursued.

\section{PREDICTORS OF DIABETES OF THE EXOCRINE PANCREAS}

\section{Severity}

The data have been mixed on whether severity of an AP episode increases the risk of developing $\mathrm{T}_{3} \mathrm{cDM}$. We know historically from the data surrounding pancreatectomies that patients with DM prior to surgery often have worsening of their disease post-operatively as greater tissue loss occurs [52]. Similarly, for recurrent AP, one study evaluated computed tomography evidence of pancreatic volume loss in patients with a single episode of AP compared with recurrent pancreatitis. The investigators found total pancreatic volume was significantly reduced in those with recurrent AP and these patients also had a strong association with endocrine and exocrine insufficiency [53].

This, and other data, suggests that the theory of greater islet cell loss, leads to greater risk of developing $\mathrm{T}_{3} \mathrm{CDM}$ and impaired glucose metabolism. This has been supported by several studies $[16,18,21-23]$. Of note, some of these studies have predominantly severe cases, while others have a majority of mild cases, and often direct cohort comparison was difficult. The meta-analysis, however, was able to compare larger cohorts of severe 
AP and mild AP and found an incidence of DM of $39 \%$ compared to $14 \%$, respectively [18].

Other studies, however, have shown no relationship between severity of AP and development of $\mathrm{T}_{3} \mathrm{CDM}$ $[17,24,51]$. These studies conclude that a mechanism, other than pure necrosis and cell loss, is at play. There are certainly limitations to these studies. In particular the meta-analysis [17] again included many studies with only severe cases of AP and was not able to do a sub-analysis based on severity of disease.

It is important to mention there has been an evolution in the classification of severity of AP: from Ranson's criteria, to APACHE II, to Balthazar score, to the Atlanta classification of AP and BISAP score, to more recently revised Atlanta criteria [4]. Many of the studies reviewed used the Atlanta criteria to classify severity; however, others used the APACHE score, and others incorporated computed tomography data (Balthazar score) to assess pancreatic necrosis. This may have led to an inability to directly compare these studies and draw broad conclusions.

Given the evidence and data collected so far, it is very likely that severity of AP and total islet cell destruction and loss plays a part in the pathophysiology of $\mathrm{T}_{3} \mathrm{cDM}$; however, it is also likely that this is not the sole risk factor or mechanism at play.

\section{Etiology}

Several studies examined etiology of pancreatitis and risk of developing $\mathrm{T}_{3} \mathrm{CDM}$. It is well established that the three most common causes of AP are: gallstone, alcohol, and hypertriglyceridemia [4]. Several studies found that alcohol was associated with greater risk of developing T3cDM $[18,19]$. These studies postulate that alcohol's effect on the pancreas directly and via its metabolites leads to multiple pathways of damage ultimately leading to atrophy, fibrosis, and premature activation of digestive enzymes. Furthermore, the specific activation of pancreatic stellate cells (by metabolites) leads to ongoing inflammatory response, fibrosis, and damage after the initial insult occurs [18]. Others, however, have found no significant association with etiology and development of T3cDM $[16,17,22]$ suggesting confounding variables exist with alcoholic pancreatitis and development of $\mathrm{T}_{3} \mathrm{CDM}$.

\section{Other risk factors}

There has been limited exploration of other risk factors associated with increased endocrine dysfunction after AP. One study explored a predictive model for developing diabetes post-AP and created a nomogram [54]. The investigators found that body mass index, age, glucose, triglycerides and low-density lipoprotein at time of admission were associated with increased risk of DM over a 3-month follow-up period. This study highlights other comorbid conditions that may contribute to worsened impaired glucose metabolism after an AP episode. Another smaller study monitored patients up to 3.5 years after AP and found that obesity and hyperlipidemia were risk factors [55]. For these risk factors it is difficult to distinguish between traditional risk factors for DM and their novel impact on T3cDM after AP. The studies conducted so far did not have control groups to distinguish natural progression to DM compared to development of DM after AP. Though the examination of risk factors for developing $\mathrm{T} 3 \mathrm{CDM}$ is limited, it may begin to highlight particular patient populations who warrant closer follow-up after an AP episode.

\section{CONCOMITANT ENDOCRINE AND EXOCRINE INSUFFICIENCY}

Many studies explored both endocrine and exocrine impairment after AP, and some found significant overlap $[12,23,56]$. Some studies cite as high as $40 \%$ overlap [56] where as others have as low as $3 \%$ overlap [19]. Many of these studies used FE1 to measure pancreatic exocrine insufficiency; however, others used need for pancreatic enzyme replacement therapy [23] and a meta-analysis used a variety of measures (secretin-caerulein infusion testing, serum pancreolauryl testing, fecal elastase and fecal fat testing, self-reported need for enzyme replacement) [56].

For chronic pancreatitis, FE1 is a commonly used indirect measure of pancreatic exocrine function. For the diagnosis of chronic pancreatitis, it has increased sensitivity with increased severity of disease $(63 \%$ mild, $100 \%$ moderate, $100 \%$ severe) and specificity of $93 \%[57,58]$. FE1 has been subject to criticism as a test for exocrine function. Specifically, it is thought it is a useful tool in ruling out pancreatic exocrine insufficiency when you have a 
low pre-test probability, however, often leads to many false positives [59].

Furthermore, some definitions of $\mathrm{T}_{3} \mathrm{CDM}$ have even included the need for evidence of exocrine dysfunction [14]. It is therefore important to characterize the relationship between endocrine and exocrine dysfunction in patients after AP and to determine the best marker for disease overlap.

\section{SCREENING RECOMMENDATIONS}

There is no consensus on when or who to screen for impaired glucose metabolism after AP. One can extrapolate from the chronic pancreatitis consensus guidelines and consider screening yearly with either fasting glucose or HbAlc [23,6o]. Additionally, it is recommended to pay particularly close attention to those with recurrent episodes or severe episodes [23]. This proposed follow-up timeline will likely capture a significant number of patients, and will avoid premature capture of the cohort who experience transient hyperglycemia following AP. There are groups who may warrant closer follow-up, namely, those with severe episodes or recurrent episodes [23]. It appears, however, that as an increasing proportion of patients develop DM at 5 years, there must be a balance between capturing patients post-event versus general population screening for DM. More research must be collected on this timeline, and what particular risk factors predispose an individual to developing T3cDM.

\section{DIABETES AS AN ETIOLOGY FOR ACUTE PAN- CREATITIS?}

A less well-established and more controversial concept that is important to mention is the bidirectional relationship between AP and diabetes. It is established that AP leads to DM; however, the reverse is less well studied.

One study examined this, using population level data derived from the Taiwan National Health Insurance claims database. The investigators first looked at the risk of developing AP in those with DM and compared those to controls. They found an increased HR of 1.72 of developing AP in those who were diabetic, and this was even higher if they had a history of "hyperglycemic crisis" (HR, 6.32). This study also found a similar relationship between developing DM after AP that many other studies have found (HR, 2.15) [24]. This study proposed that given the higher HR in those with a history of hyperglycemic crisis, there might be a "severity-response" relationship. Several mechanisms were proposed in this study including: (1) chronic hyperglycemia leads to increased reactive oxygen species, increased lipid peroxidase which may lead to AP episodes; (2) association with comorbid conditions such as obesity, hyperlipidemia, and gallstones which can precipitate AP; (3) cellular mechanisms including enhanced ryanodine receptor function leading to alterations in cellular mechanisms, specifically calcium and is a similar pathway involved in AP and DM [24]. Other studies have also supported this association. One study in particular showed a HR of 1.49 even after controlling for common comorbidities such as age, gender, obesity, smoking, alcohol use, or gallbladder disease [61].

Another study approached this question by examining the structural changes that occur in the pancreas as a result of DM [62]. They found pancreatic weight and volume were decreased in those with $\operatorname{TiDM}$ (no significant decrease in $\mathrm{T} 2 \mathrm{DM}$ ), and at autopsy, the investigators found fibrosis with minimal inflammatory changes and no duct abnormalities in these patients. Additionally, these patients were largely asymptomatic, despite having reduced FE1 levels. This study highlights a disease entity separate from chronic pancreatitis. This suggests pancreatic fibrosis and exocrine dysfunction exists separately (or on a continuum) from chronic pancreatitis and occurs most predominantly in those with TiDM. Though this cohort did not develop AP episodes, this study does highlight the presence of structural changes within the pancreas that may increase a patient's risk for developing AP, further showing the complex interplay between the endocrine and exocrine pancreas.

\section{CONCLUSIONS}

In conclusion, DM (including $\mathrm{T}_{3} \mathrm{CDM}$ ) and impaired glucose metabolism is common and increasingly recognized following AP. Among the types of DM, T3cDM is an increasingly recognized entity and has been found 
following AP. Though the diagnostic criteria have varied over time and it is largely underrecognized, its unique disease profile warrants further attention. These patients typically require insulin earlier than those with T2DM and often have difficult to manage hypo- and hyperglycemic episodes. Several large studies estimate prevalence of about $15 \%$ at 1 year and even greater proportion of cases at 5 years. Severity appears to affect propensity of developing diabetes, and several studies have found that alcohol may also be correlated. Physicians should be aware and aim to screen patients yearly following AP episode, and pay particular attention to those with severe episodes, alcoholic pancreatitis, and diabetes risk factors. Finally, there are data suggesting diabetes leads to structural changes in the pancreas potentially predisposing to AP, further highlighting the complex interplay between AP and the endocrine pancreas. This review highlights that diabetes following AP is an increasingly recognized clinical entity; however, currently the data are limited and heterogeneous and future studies are needed to clarify the existing gaps in knowledge.

\section{Conflict of interest}

No potential conflict of interest relevant to this article was reported.

\section{REFERENCES}

1. Krishna SG, Kamboj AK, Hart PA, Hinton A, Conwell DL. The changing epidemiology of acute pancreatitis hospitalizations: a decade of trends and the impact of chronic pancreatitis. Pancreas 2017;46:482-488.

2. Yadav D, Lowenfels AB. The epidemiology of pancreatitis and pancreatic cancer. Gastroenterology 2013;144:12521261.

3. Peery AF, Crockett SD, Barritt AS, et al. Burden of gastrointestinal, liver, and pancreatic diseases in the United States. Gastroenterology 2015;149:1731-1741.

4. Forsmark ChE, Vege SS, Wilcox CM. Acute pancreatitis. N Engl J Med 2017;376:598-599.

5. Roglic G; World Health Organization. Global Report on Diabetes. Geneva (CH): World Health Organization, 2016.

6. Ewald N, Kaufmann C, Raspe A, Kloer HU, Bretzel RG, Hardt PD. Prevalence of diabetes mellitus secondary to pancreatic diseases (type 3c). Diabetes Metab Res Rev
2012;28:338-342.

7. Woodmansey C, McGovern AP, McCullough KA, et al. Incidence, demographics, and clinical characteristics of diabetes of the exocrine pancreas (type $3 c$ ): a retrospective cohort study. Diabetes Care 2017;40:1486-1493.

8. Cui Y, Andersen DK. Pancreatogenic diabetes: special considerations for management. Pancreatology 2011;11:279-294.

9. Hart PA, Bellin MD, Andersen DK, et al. Type зc (pancreatogenic) diabetes mellitus secondary to chronic pancreatitis and pancreatic cancer. Lancet Gastroenterol Hepatol 2016;1:226-237.

10. Pannala R, Leirness JB, Bamlet WR, Basu A, Petersen GM, Chari ST. Prevalence and clinical profile of pancreatic cancer-associated diabetes mellitus. Gastroenterology 2008;134:981-987.

11. Wynne K, Devereaux B, Dornhorst A. Diabetes of the exocrine pancreas. J Gastroenterol Hepatol 2019;34:346-354.

12. Nikkola J, Laukkarinen J, Lahtela J, et al. The long-term prospective follow-up of pancreatic function after the first episode of acute alcoholic pancreatitis: recurrence predisposes one to pancreatic dysfunction and pancreatogenic diabetes. J Clin Gastroenterol 2017;51:183-190.

13. American Diabetes Association. 2. Classification and diagnosis of diabetes: standards of medical care in diabetes-2018. Diabetes Care 2018;41(Suppl 1):S13-S27.

14. Ewald N, Bretzel RG. Diabetes mellitus secondary to pancreatic diseases (type 3c): are we neglecting an important disease? Eur J Intern Med 2013;24:203-206.

15. Roeyen G, De Block C. A plea for more practical and clinically applicable criteria defining type $3 c$ diabetes. Pancreatology 2017;17:875.

16. Andersson B, Pendse ML, Andersson R. Pancreatic function, quality of life and costs at long-term follow-up after acute pancreatitis. World J Gastroenterol 2010;16:49444951.

17. Das SL, Singh PP, Phillips AR, Murphy R, Windsor JA, Petrov MS. Newly diagnosed diabetes mellitus after acute pancreatitis: a systematic review and meta-analysis. Gut 2014;63:818-831.

18. Zhi M, Zhu X, Lugea A, Waldron RT, Pandol SJ, Li L. Incidence of new onset diabetes mellitus secondary to acute pancreatitis: a systematic review and meta-analysis. Front Physiol 2019;10:637.

19. Ho TW, Wu JM, Kuo TC, et al. Change of both endocrine and exocrine insufficiencies after acute pancreatitis in 
non-diabetic patients: a nationwide population-based study. Medicine (Baltimore) 2015;94:e1123.

20. Umapathy C, Raina A, Saligram S, et al. Natural history after acute necrotizing pancreatitis: a large US tertiary care experience. J Gastrointest Surg 2016;20:1844-1853.

21. Tu J, Yang Y, Zhang J, et al. Effect of the disease severity on the risk of developing new-onset diabetes after acute pancreatitis. Medicine (Baltimore) 2018;97:e10713.

22. Vujasinovic M, Tepes B, Makuc J, et al. Pancreatic exocrine insufficiency, diabetes mellitus and serum nutritional markers after acute pancreatitis. World J Gastroenterol 2014;20:18432-18438.

23. Vipperla K, Papachristou GI, Slivka A, Whitcomb DC, Yadav D. Risk of new-onset diabetes is determined by severity of acute pancreatitis. Pancreas 2016;45:e14-e15.

24. Lee YK, Huang MY, Hsu CY, Su YC. Bidirectional relationship between diabetes and acute pancreatitis: a population-based cohort study in Taiwan. Medicine (Baltimore) 2016;95:e2448.

25. Johansen K, Ornsholt J. Frequency of diabetes after acute pancreatitis. Metabolism 1972;21:291-296.

26. Olszewski S, Kinalska I, Dlugosz J, Stasiewicz J, Gabryelewicz A. The glucose tolerance, insulin response and pancreatic exocrine function in patients after acute pancreatitis. Endokrinologie 1978;71:183-191.

27. Seligson U, Ihre T, Lundh G. Prognosis in acute haemorrhagic, necrotizing pancreatitis. Acta Chir Scand 1982;148:423-429.

28. Angelini G, Pederzoli P, Caliari S, et al. Long-term outcome of acute necrohemorrhagic pancreatitis. A 4-year follow-up. Digestion 1984;30:131-137.

29. Eriksson J, Doepel M, Widen E, et al. Pancreatic surgery, not pancreatitis, is the primary cause of diabetes after acute fulminant pancreatitis. Gut 1992;33:843-847.

30. Doepel M, Eriksson J, Halme L, Kumpulainen T, Hockerstedt K. Good long-term results in patients surviving severe acute pancreatitis. Br J Surg 1993;80:1583-1586.

31. Angelini G, Cavallini G, Pederzoli P, et al. Long-term outcome of acute pancreatitis: a prospective study with 118 patients. Digestion 1993;54:143-147.

32. Malecka-Panas E, Juszynski A, Wilamski E. Acute alcoholic pancreatitis does not lead to complete recovery. Mater Med Pol 1996;28:64-68.

33. Appelros S, Lindgren S, Borgstrom A. Short and long term outcome of severe acute pancreatitis. Eur J Surg 2001;167:281-286.
34. Malecka-Panas E, Gasiorowska A, Kropiwnicka A, Zlobinska A, Drzewoski J. Endocrine pancreatic function in patients after acute pancreatitis. Hepatogastroenterology 2002;49:1707-1712.

35. Ibars EP, Sanchez de Rojas EA, Quereda LA, Ramis RF, Sanjuan VM, Peris RT. Pancreatic function after acute biliary pancreatitis: does it change? World J Surg 2002;26:479-486.

36. Halonen KI, Pettila V, Leppaniemi AK, Kemppainen EA, Puolakkainen PA, Haapiainen RK. Long-term health-related quality of life in survivors of severe acute pancreatitis. Intensive Care Med 2003;29:782-786.

37. Boreham B, Ammori BJ. A prospective evaluation of pancreatic exocrine function in patients with acute pancreatitis: correlation with extent of necrosis and pancreatic endocrine insufficiency. Pancreatology 2003;3:303-308.

38. Szentkereszty Z, Agnes C, Kotan R, et al. Quality of life following acute necrotizing pancreatitis. Hepatogastroenterology 2004;51:1172-1174.

39. Hochman D, Louie B, Bailey R. Determination of patient quality of life following severe acute pancreatitis. Can J Surg 2006;49:101-106.

40. Kaya E, Dervisoglu A, Polat C. Evaluation of diagnostic findings and scoring systems in outcome prediction in acute pancreatitis. World J Gastroenterol 2007;13:30903094.

41. Yasuda T, Ueda T, Takeyama Y, et al. Long-term outcome of severe acute pancreatitis. J Hepatobiliary Pancreat Surg 2008;15:397-402.

42. Gupta R, Wig JD, Bhasin DK, et al. Severe acute pancreatitis: the life after. J Gastrointest Surg 2009;13:1328-1336.

43. Pelli H, Lappalainen-Lehto R, Piironen A, Jarvinen S, Sand J, Nordback I. Pancreatic damage after the first episode of acute alcoholic pancreatitis and its association with the later recurrence rate. Pancreatology 2009;9:245251.

44. Uomo G, Gallucci F, Madrid E, Miraglia S, Manes G, Rabitti PG. Pancreatic functional impairment following acute necrotizing pancreatitis: long-term outcome of a non-surgically treated series. Dig Liver Dis 2010;42:149152.

45. Garip G, Sarandol E, Kaya E. Effects of disease severity and necrosis on pancreatic dysfunction after acute pancreatitis. World J Gastroenterol 2013;19:8065-8070.

46. Chandrasekaran P, Gupta R, Shenvi S, et al. Prospective comparison of long term outcomes in patients with se- 
vere acute pancreatitis managed by operative and non operative measures. Pancreatology 2015;15:478-484.

47. Winter Gasparoto RC, Racy Mde C, De Campos T. Longterm outcomes after acute necrotizing pancreatitis: what happens to the pancreas and to the patient? JOP 2015;16:159-166.

48. Tu J, Zhang J, Ke L, et al. Endocrine and exocrine pancreatic insufficiency after acute pancreatitis: long-term follow-up study. BMC Gastroenterol 2017;17:114.

49. Plummer MP, Finnis ME, Phillips LK, et al. Stress induced hyperglycemia and the subsequent risk of type 2 diabetes in survivors of critical illness. PLoS One 2016;11:e0165923.

50. McCowen KC, Malhotra A, Bistrian BR. Stress-induced hyperglycemia. Crit Care Clin 2001;17:107-124.

51. Shen HN, Yang CC, Chang YH, Lu CL, Li CY. Risk of diabetes mellitus after first-attack acute pancreatitis: a national population-based study. Am J Gastroenterol 2015;110:1698-1706.

52. Burkhart RA, Gerber SM, Tholey RM, et al. Incidence and severity of pancreatogenic diabetes after pancreatic resection. J Gastrointest Surg 2015;19:217-225.

53. Avanesov M, Loser A, Smagarynska A, et al. Clinico-radiological comparison and short-term prognosis of single acute pancreatitis and recurrent acute pancreatitis including pancreatic volumetry. PLoS One 2018;13:e0206062.

54. Ma JH, Yuan YJ, Lin SH, Pan JY. Nomogram for predicting diabetes mellitus after the first attack of acute pancreatitis. Eur J Gastroenterol Hepatol 2019;31:323-328.

55. Wu D, Xu Y, Zeng Y, Wang X. Endocrine pancreatic function changes after acute pancreatitis. Pancreas 2011;40:1006-1011.

56. Das SL, Kennedy JI, Murphy R, Phillips AR, Windsor JA, Petrov MS. Relationship between the exocrine and endocrine pancreas after acute pancreatitis. World J Gastroenterol 2014;20:17196-17205.

57. Loser C, Mollgaard A, Folsch UR. Faecal elastase 1: a novel, highly sensitive, and specific tubeless pancreatic function test. Gut 1996;39:580-586.

58. Dominguez-Munoz JE, Hieronymus C, Sauerbruch T, Malfertheiner P. Fecal elastase test: evaluation of a new noninvasive pancreatic function test. Am J Gastroenterol 1995;90:1834-1837.

59. Vanga RR, Tansel A, Sidiq S, El-Serag HB, Othman MO. Diagnostic performance of measurement of fecal elastase-1 in detection of exocrine pancreatic insufficiency: systematic review and meta-analysis. Clin Gastroenterol Hepatol 2018;16:1220-1228.

6o. Rickels MR, Bellin M, Toledo FG, et al. Detection, evaluation and treatment of diabetes mellitus in chronic pancreatitis: recommendations from PancreasFest 2012. Pancreatology 2013;13:336-342.

61. Girman CJ, Kou TD, Cai B, et al. Patients with type 2 diabetes mellitus have higher risk for acute pancreatitis compared with those without diabetes. Diabetes Obes Metab 2010;12:766-771.

62. Mohapatra S, Majumder S, Smyrk TC, et al. Diabetes mellitus is associated with an exocrine pancreatopathy: conclusions from a review of literature. Pancreas 2016;45:11041110. 